\title{
Study of deuterium metal interaction by ion implantation
}

\author{
B PANIGRAHI, K G M NAIR and KANWAR KRISHAN \\ Materials Science Division, Indira Gandhi Centre for Atomic Research, Kalpakkam 603 102, \\ India

\begin{abstract}
Dynamic and non-equilibrium effects involving interaction between deuterium and radiation produced defects were studied during deuterium implantation of $\mathrm{Cu}$ and $\mathrm{Ti}$. The technique of neutron yield measurement during deuterium implantation was employed and theoretical analysis was made to study the dynamics. SIMS, GIXRD and SEM studies on deuterium implanted samples were employed to study the evolution of implanted deuterium profiles, structure of deuterides and surface topography respectively.
\end{abstract}

Keywords. Deuterium; implantation; deuteride; diffusion; titanium hydride; copper.

\section{Introduction}

The behaviour of hydrogen and its isotopes has evoked considerable interest from both fundamental and technological points of view (Myers et al 1992). Hydrogen-metal systems have found diverse applications such as in fuel cells and hydrogen energy systems. Further in the plasma environments of Tokamak fusion reactors (Winter $e t$ al 1989), hydrogen metal chemical reactions are augmented by athermal implantation of $\mathrm{H}$ directly into meta! matrix making $\mathrm{H}$-defect interaction in metals a critical issue in these applications. Hence hydrogen behaviour in metals has been extensively investigated and a large volume of data exist on the diffusion, phase diagram, solubility and surface adsorption in metal-hydrogen systems. However, most of these studies are performed after loading the sample with hydrogen via high pressure gas charging, electrolysis etc and hence pertain to static equilibrium properties. New and interesting effects come into play when one considers dynamic and non-equilibrium effects involving the interaction between the hydrogen and various mobile defects as would happen during implantation of deuterium into metals. The dynamical and nonequilibrium effects that come into play during implantation of deuterium into metals have not been studied extensively. This is largely due to the difficulties in designing and carrying out in-situ experiments. We have investigated the dynamics by measuring the time dependent neutron yield due to $d(d, n)^{3} \mathrm{He}$ reaction during $30 \mathrm{keV}$ deuterium implantation of metals. Comparison of the nuetron yield data with theoretical analysis of neutron yield behaviour provided information on the dynamics and non-linear behaviour during implantation. We have chosen $\mathrm{Cu}$ and Ti metals for this study having widely differing properties with respect to hydrogen and its isotopes especially with respect to stability of their hydrides, hydrogen defect interaction and hydrogen diffusion.

Apart from the study of dynamics, deuterium implanted metal samples provide interesting hydrogen metal system for study. As in these systems considerable amount of displacement damage and strain is also produced due to implantation, the structure and kinetics of deuteride phase formation in these systems are likely to be different in comparison to the thermal equilibrium conditions. We have used secondary ion mass spectrometry (SIMS), glancing incidence X-ray diffraction (GIXRD) and scanning 
electron microscopy (SEM) techniques on deuterium implanted samples to study the structure and kinetics of deuteride phase formation in $\mathrm{Ti}$ and the deuterium dynamics at the subsurface layers in $\mathrm{Cu}$. In this paper we give a brief report of our work.

\section{Study of dynamics by neutron yield experiment}

The experimental arrangement is schematically depicted in figure 1. A deuterium beam of constant energy and current is incident on the metal and the neutron yield due to the $d(d, n)^{3} \mathrm{He}$ reaction is measured as a function of time. The deuterium is implanted at a depth $R$ which is the range of ions with a stragling of $\Delta R$. However, as the deuterium is quite mobile, it gradually builds a time dependent concentration profile $C(x, t)$. The exact form of the profile at any time is decided by different solid state processes such as defect reactions involving the implanted deuterium atoms and the radiation damage processes, desorption from surface, formation of metal deuteride and deuterium gas bubbles. The implanted deuterium reacts with the incident deuterium atoms via $d(d, n)^{3} \mathrm{He}$ reaction. As the incident deuterium atoms degrade in energy, their mean energy at a depth $x$ will be given by $E(x)$. If the cross section for this reaction at a depth $x$ is $\sigma(E(x)$ ), then for an incident beam current $I$ on a foil of thickness $h$ and area $A$, we get the neutron yield $N(m T)$ in the time interval $m T$ to $(m+1) T$ to be

$$
N(m, T)=A J \int_{0}^{h} \mathrm{~d} x \sigma(E(x)) \int_{m T}^{(m+1) T} C(x, t) \mathrm{d} t .
$$

The cross section $\sigma(E(x))$ decreases very rapidly with $E(x)$ (see figure 2), so the dominant contribution to the integral comes from the concentration profile $C(x, t)$ close to the surface, which is indicated by the shaded area in figure 2 . Hence the evolution of neutron yieid as a function of time contains information about the time evolution of deuterium concentration profile $C(x, t)$. Since $C(x, t)$ is governed by different solid state processes, information on these are obtained by comparing the model calculations with the neutron yield data.

\subsection{Modelling of neutron yield during implantation}

The concentration profile of deuterium is obtained by solving the diffusion equation with the implantation source term and appropriate boundary condition. Using this, the

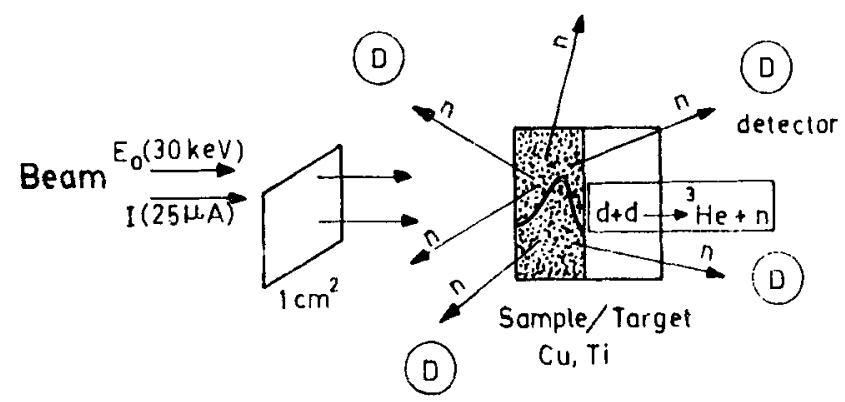

Figure 1. Schematic of the experimental arrangement of neutron yield experiment. 


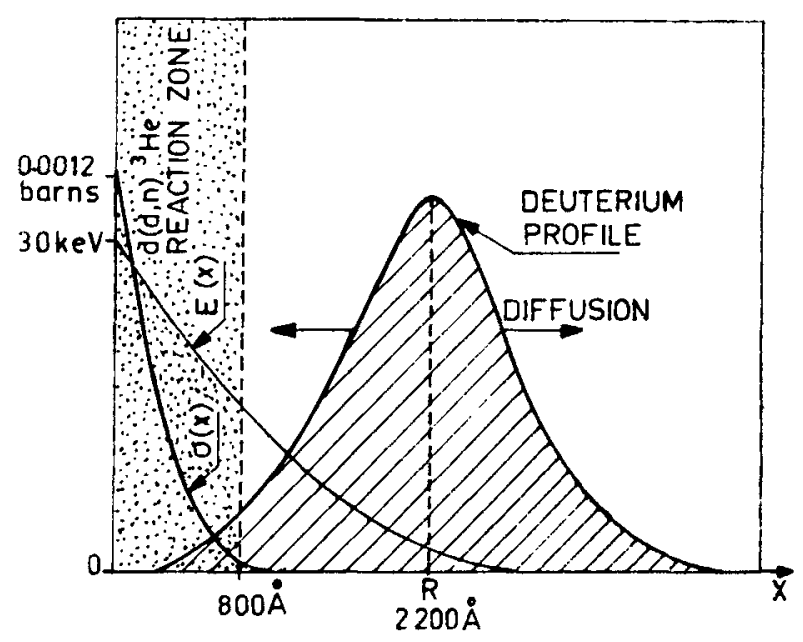

Figure 2. Schematic presentation of the implantation profile, energy of the $30 \mathrm{keVD}^{+}$beam and the corjesponding $d(d, n)^{3} \mathrm{He}$ cross-section as a function of depth in the sample. The region of the sample from which most of the neutrons are expected to be emitted is shown as the dotted region in the figure.

neutron yield during implantation of deuterium is evaluated from the knowledge of stopping power and $d-d$ cross-section data.

We have obtained a numerical expression for the concentration profile of deuterium by solving the diffusion equation with desorption boundary condition which was used to evaluate the concentration profile of deuterium during implantation of $\mathrm{Cu}$. Since during implantation condition deuterium atoms are introduced concurrently with displacement damage, deuterium migration is influenced by reaction of deuterium atoms with irradiation produced defects which are themselves evolving in time. We have used the effective diffusion approach (Mansur et al 1986). Details of the method of calculation and the resulting expressions involving multiple integrals were reported elsewhere (Panigrahi et al 1992). Using the time dependent concentration profiles we have estimated the neutron yield as a function of time with diffusion constant as a parameter and comparing this with the experimental data we have determined the diffusion constant during implantation of deuterium in $\mathrm{Cu}$.

We have also analytically examined the asymptotic behaviour of neutron yield. The cross-section $\sigma(E)$ as a function of energy has been measured by many workers (Krauss et al 1987) and in the energy range $13 \mathrm{keV}$ to $30 \mathrm{keV}$ a good approximation is

$$
\sigma(E)=1 \cdot 18 \times 10^{-9} E^{4} \text { barn; }(E \text { in } \mathrm{keV}) .
$$

An analytical form of $E(x)$ can be quite easily obtained from the Lindhard model (Lindhard and Scharff 1961) which gives the rate of energy loss to be proportional to the square root of the energy i.e. $\mathrm{d} E / \mathrm{d} x=k E^{1 / 2}$. Using this we get

$$
\sigma(x)=1 \cdot 18 \times 10^{-9} E_{0}^{4}\left(1-\frac{x}{R}\right)^{8} ; \quad E_{0} \text { in } \mathrm{keV}
$$

where $E_{0}$ is the energy of the deuterons as it enters the surface and $R$ the range. 
Table 1. Theoretical estimates of the exponent $b$ for different model calculations.

\begin{tabular}{ll}
\hline Model & $b$ \\
\hline $\begin{array}{l}\text { No diffusion } \\
\text { Free diffusion }\end{array}$ & 2 \\
$\begin{array}{l}\text { Diffusion and desorption } \\
\text { Diffusion hindered by } \\
\text { formation of hydrides }\end{array}$ & $1 \cdot 5$ \\
\hline
\end{tabular}

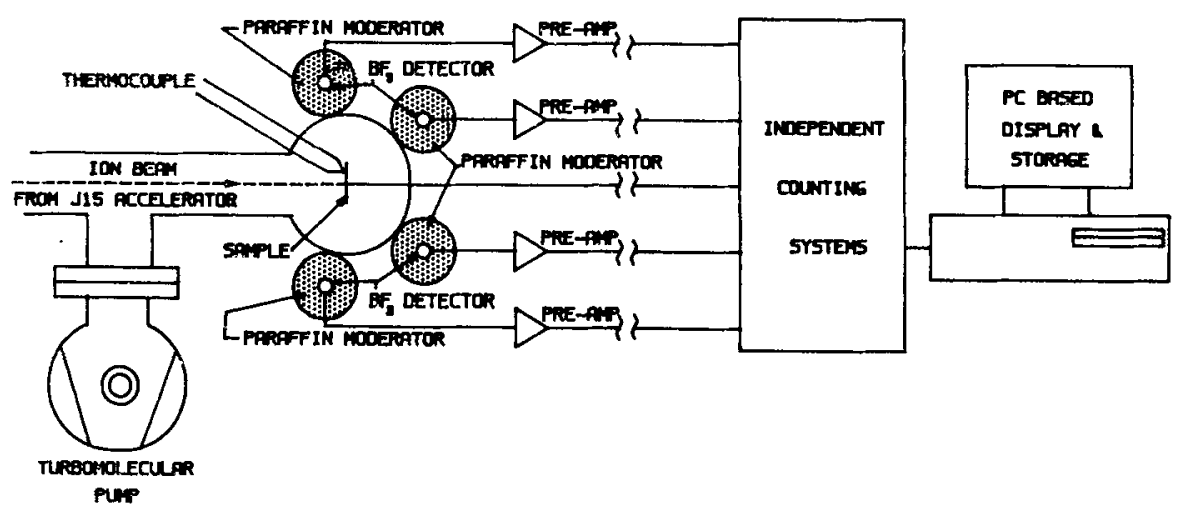

Figure 3. The details of the implantation set up, showing schematically the implantation chamber, the neutron detectors and the counting electronics.

A detailed calculation of the concentration profile $C(x, t)$, incorporating effects like diffusion, desorption, deuteride formation is given elsewhere (Sahu et al 1992).

From the above analysis one can easily get some idea about the nature of the time dependent neutron yield spectrum. For example, if there is no diffusion then $C(x, t)$ will be directly proportional to $t$ and hence from (1) we observe that $N(m, T) \propto(2 m+1) T^{2}$. In general, however, $N(t=m T)$ and the total number of neutrons produced up to time $t$ will behave like $a t^{b}$, where $a$ and $b$ are constants. The value of exponents for different processes are tabulated in table 1 . These exponents are compared with the experimentally measured exponents to obtain information on the dominant solid state process occurring during implantation of $\mathrm{Cu}$ and $\mathrm{Ti}$.

\subsection{Experimental}

The deuterium implantation have been carried out using a Sames Model J-15 accelerator. Figure 3 gives a schematic of this set up. The neutrons produced by $d(d, n)^{3} \mathrm{He}$ reaction are detected by four boron lined detectors placed outside the sample chamber and the counts are measured by four independent counting channels. The counting and recording of the neutrons are automated using a personal computer. The $\mathrm{Cu}$ samples used in the work had a nominal purity of $99.99 \%$ and was annealed at $850^{\circ} \mathrm{C}$ for $2 \mathrm{~h}$. The Ti samples used had a nominal purity of $99 \cdot 6+\%$. All the samples are polished 
using standard metallographic techniques. Details of the experimental set up and conditions are given by Panigrahi (1993).

\subsection{Results and discussion}

The cumulative neutron counts, i.e., the total counts recorded up to time $t$, for $\mathrm{Cu}$ and Ti for room temperature implantation is shown in figure 4, which shows a monotonic increase in time. The data were fitted to a power law and the exponents $b$ obtained are listed in table 2. Comparing the experimental values of exponent $b$ with the model results (table 1), we make the following inferences regarding the solid state process occurring during implantation.

The experimental value of $b$ in $\mathrm{Cu}$ at room temperature is 1.16 which is close to the theoretical value 1.0 for the case when diffusion followed by desorption determines the concentration profile. Further by comparing (see figure 5) the time dependence of the calculated neutron yield for different values of diffusion constant with the time dependence of observed neutron yield data we obtain a diffusion constant of $5 \times 10^{-13} \mathrm{~cm}^{2} / \mathrm{sec}$ which is close to the value of vacancy diffusion constant in $\mathrm{Cu}$. Since, deuterium vacancy complexes are stable at the temperature of irradiation (Lengler $e t$ al 1978 ) and vacancy diffusion constant is around $5 \times 10^{-13} \mathrm{~cm}^{2} / \mathrm{sec}$ (Panigrahi 1993), we attribute the deuterium diffusion in $\mathrm{Cu}$ during implantation to a vacancy mediated diffusion of trapped deuterium.

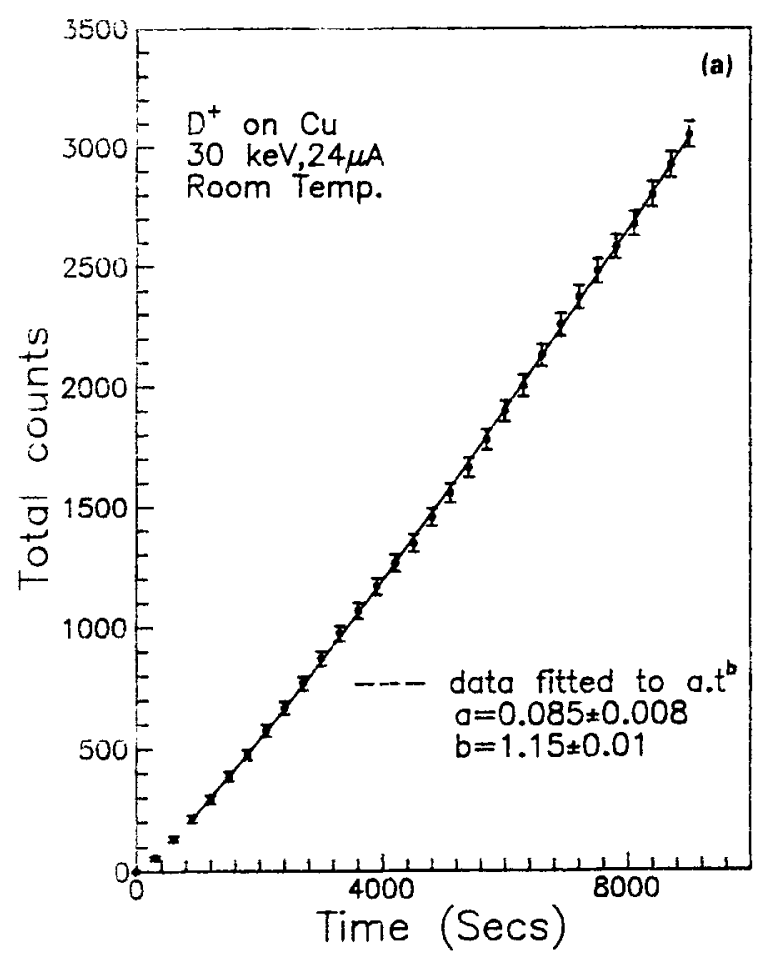

Nigure 4. 


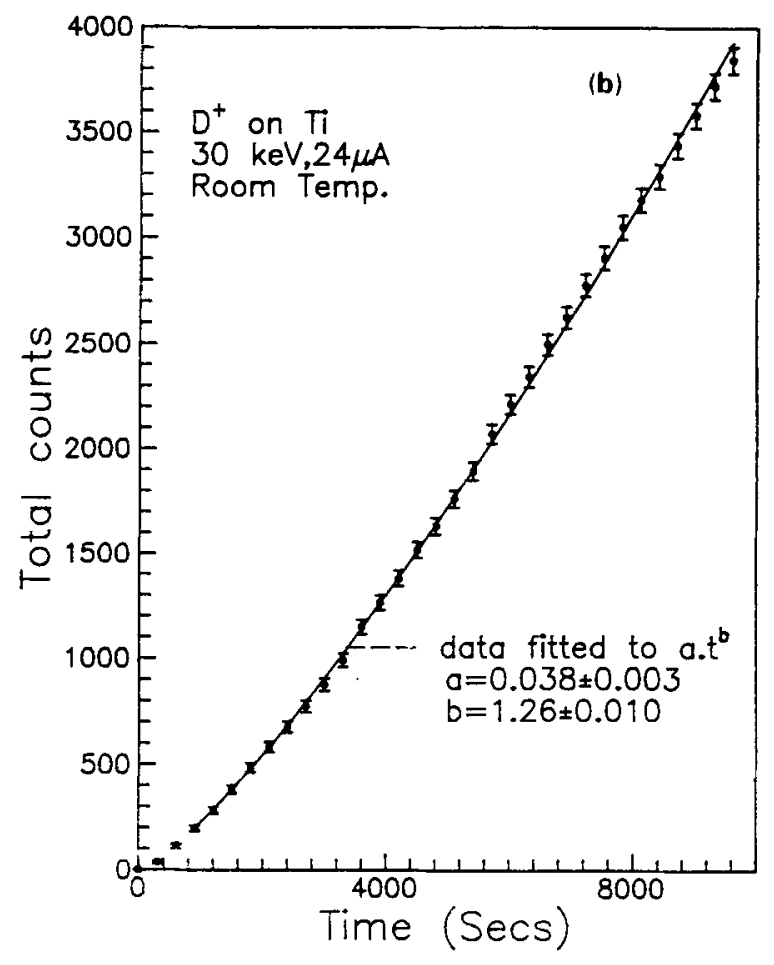

Figure 4. The cumulative neutron counts in $\mathrm{Cu}(\mathbf{a})$ and $\mathrm{Ti}$ (b) as a function of time. The data has been fitted to a power law of the form $t^{b}$.

Table 2. Experimental values of exponent $b$ for different metals at room temperature and low temperatures.

\begin{tabular}{lll}
\hline Metal & Room temp. & Low temp. \\
\hline $\mathrm{Cu}$ & $1 \cdot 16$ & $1.37(T=130 \mathrm{~K})$ \\
$\mathrm{Ti}$ & $1 \cdot 26$ & $1.49(T=140 \mathrm{~K})$ \\
\hline
\end{tabular}

From table 2 we observe that the value of exponent observed for $\mathrm{Ti}$ at room temperature is $1 \cdot 26$. This is a surprising result for $\mathrm{Ti}$, for hydrides of $\mathrm{Ti}$ are known to be stable (Mueller et al 1968) at room temperature under equilibrium conditions and deuterium concentration near the peak exceeds the solubility limit (Panigrahi 1993). If deuterides were to form during implantation, the exponent $b$ is expected to be close to 2 . The considerably lower values of $b$ observed in our experiment indicate that there is no tendency for formation of deuteride during implantation even in a narrow zone of specimen around the peak of the implantation profile, where the implanted deuterium concentration is the highest. This result is corroborated by the SIMS and by glancing incidence XRD study of deuterium implanted Ti samples reported below, where it is seen that the deuterium immediately after implantation moves towards the surface until a saturation concentration is reached at the near-surface region. Then this layer grows inwards, and at a dose of $2 \times 10^{18} \mathrm{D}^{+}$ions $/ \mathrm{cm}^{2}$, extends much beyond the range of deuterium in $\mathrm{Ti}$. Implanted samples when examined by glancing incidence XRD 


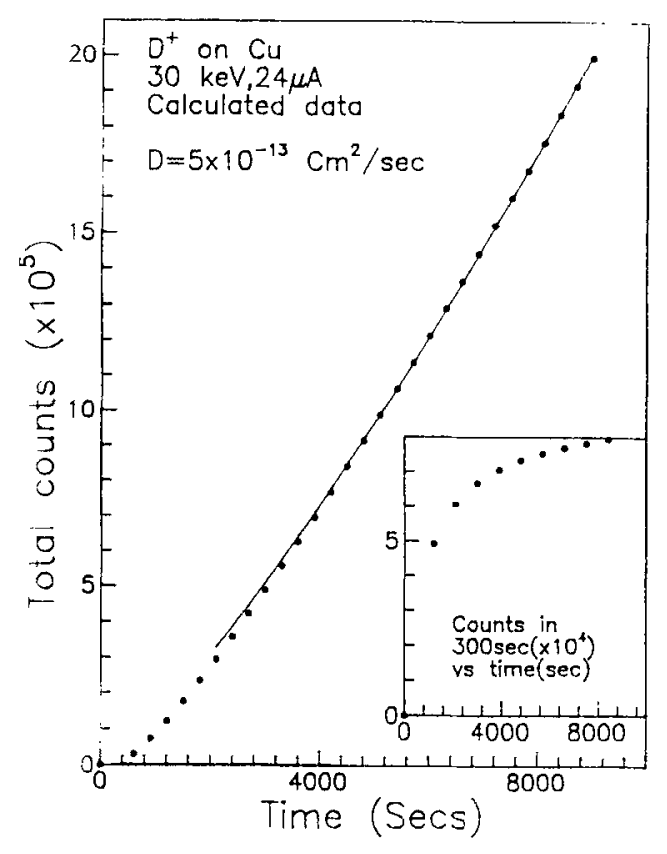

Figure 5. Veutron yield as a function of implantation time calculated using (1). The diffusion constant of deuterium was taken to be $5 \times 10^{-13} \mathrm{~cm}^{2} / \mathrm{sec}$. The value of $h$ in (1) was taken as $2594 \mathrm{~A}$, corresponding to the range of $30 \mathrm{keV} \mathrm{D}$ in $\mathrm{Cu}$. The current density $J$ was taken as $15 \times 10^{14} \mathrm{D}^{+}$ions $/ \mathrm{cm}^{2} / \mathrm{sec}$ and the area $A=1 \mathrm{~cm}^{2}$. The data was fitted to a power law. $a t^{t}$, $a=24 \cdot 37$ and $b=1 \cdot 24$. The fitted function is plotted as a solid line in the graph. The data in 1 -axis should be multiplied by the detector efficiency factor $s=8 \times 10^{-4}$ for comparison with the experimental data. The inset in the graph shows the neutron counts, observed in $300 \mathrm{sec}$. interval. as a function of implantation time.

showed the presence of titanium deuteride phase. Although we have not carried out a detailed analysis of the kinetics of this case, it appears that the deuteride layer grows inward with time and the deuterium concentration saturates at the surface. Since the time dependence of the neutron yield arises from the time dependence of the deuterium concentration at the surface, the neutron yield in the asymptotic limit should also saturate giving rise to a value of $b$ closer to 1 which is consistent with the observations.

\section{Deuteriding behaviour of titanium implanted with deuterium ions}

In this work, we study the structure and kinetics of deuteride phase formation on samples of Ti implanted with deuterium to various doses by using secondary ion mass spectrometry (SIMS) and glancing incidence X-ray diffraction (GIXRD) techniques. The implantation was carried out at room temperature using a mass analyzed UHV implantation system. The SIMS analyses were carried out in a CAMECA IMS-4f ion microprobe using a $14.5 \mathrm{keV} \mathrm{Cs}{ }^{+}$primary ion beam for sputtering and negative secondary ions for deuterium analysis. Details regarding the implantation condition and SIMS analysis are given by Panigrahi et al (1994). Glancing angle X-ray diffraction technique has been used to investigate the nature of hydride formed in the near surface region of deuterium implanted titanium samples. The geometry of the X-ray diffraction 


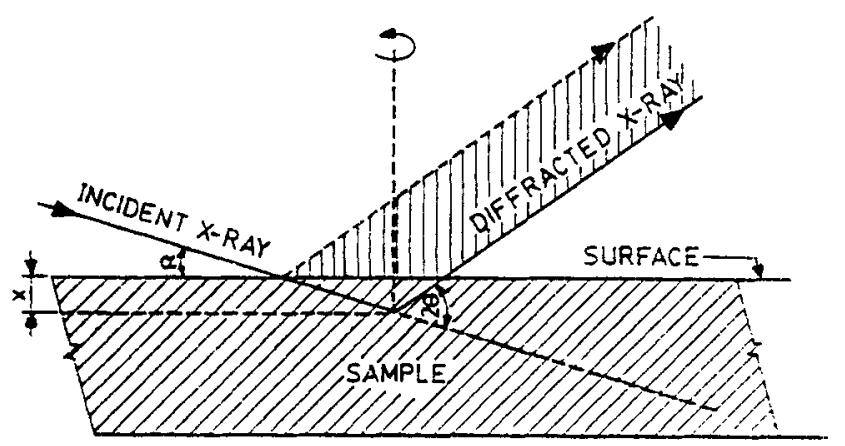

Figure 6. The scattering geometry during glancing angle $X$-ray diffraction study, $x$ is the angle of incidence, 20 the diffraction angle, $X$ denotes the penetration depth of $\mathrm{X}$-rays for $99 \%$ absorption of the incident $X$-rays. The axis of rotation during spinning of the sample is also indicated in the sketch. In our experiment $X$-ray diffraction measurements are carried out using $\mathrm{CuK}_{2}$ radiation using a model D-5000 Siemens diffractometer. The angle of incidence was fixed at $5^{\prime \prime}$ which gave a probe depth $X=3.8 \mu \mathrm{m}$.

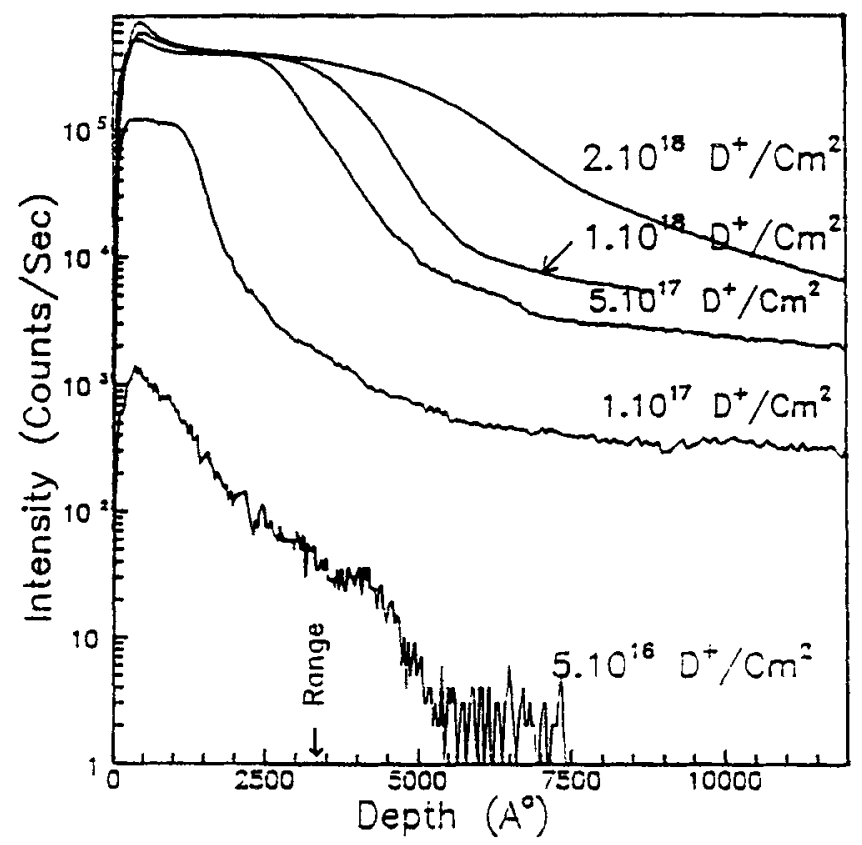

Figure 7. The depth profile of deuterium from Ti samples implanted with $60 \mathrm{keV} \mathrm{D}_{2}$ ions at room temperature to different doses. The dose rate of the impiantation was $2.6 \times 10^{14} \mathrm{D}^{+}$ ions $/ \mathrm{cm}^{2} / \mathrm{sec}$. The dose corresponding to each sample is indicated in the plot. The range of deuterium ion is $3350 \mathrm{~A}$ which is indicated by an arrow mark in the plot.

experiment is explained in figure 6 . The evolution of concentration profile of deuterium as a function of dose in the range $(0.5-20) \times 10^{17} \mathrm{D}^{+}$ions $/ \mathrm{cm}^{2}$ maintaining a constant implantation flux of $2.6 \times 10^{14} \mathrm{D}^{+}$ions $/ \mathrm{cm}^{2} / \mathrm{sec}$ is presented in figure 7 . It is seen that the deuterium moves toward the surface immediately after implantation until a saturation concentration is reached within the near surface region at dose of less than $5 \times 10^{17} \mathrm{D}^{+}$ions $/ \mathrm{cm}^{2}$ and then this layer grows inwards reaching a depth larger than 


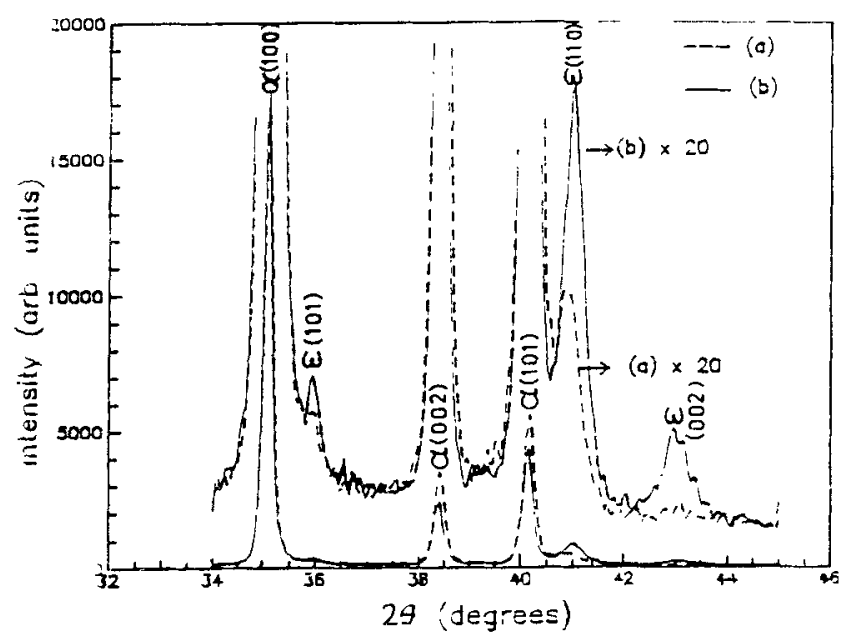

Figure 8. vilancing incidence $X$-ray data from deuterium implanted with $\mathrm{T}_{1}$ (a) to a dose of $2 \times 10^{18} \mathrm{D}^{+}$lons $/ \mathrm{cm}^{2}$. (b) $5 \times 10^{16} \mathrm{D}^{+}$ions $\mathrm{cm}^{2}$. The daia from curves a and $b$ are multiplied by 20 and plotted as curve $\mathrm{a} \times 20$ and $\mathrm{b} \times 20$ to show the weak peaks. The intensities corresponding to the $x$-Ti phase are seen to he reduced along with the build up of deuteride phase indicating the reduction of volume fraction of $x$-Ti phase with the build up of $x$-Ti deuteride phase.

$5000 \mathrm{~A}$ at a dose of $2 \times 10^{18} \mathrm{D}^{+}$ions $/ \mathrm{cm}^{2}$. It may be noted that the concentration profile observed is very different from the familiar Gaussian type implantation profile centred around the range of the deuterium ion. The saturation deuterium concentration has been estimated by equating the area under the observed $\mathrm{D}^{-}$secondary ion yield curve to the total implantation dose. The deuterium concentration obtained in atoms $/ \mathrm{cm}^{3}$ corresponds to a maximum $[\mathrm{D}] /[\mathrm{Ti}]$ atomic concentration ratio of about 1 . This clearly indicates the formation of titanium deuteride in the near-surface region.

The data obtained from GIXRD experiment is presented in figure 8, showing the first three deuteride peaks observed for sample implanted with $60 \mathrm{keV} \mathrm{D}+V_{2}$ ions to a dose of $2 \times 10^{18} \mathrm{D}^{+}$ions $/ \mathrm{cm}^{2}$. Since the penetration depth is 3.8 micron at an incident angle of $5^{\circ}$, the X-ray peaks from hydride is found along with the Ti (hcp) peaks. The X-ray data of a sample implanted up to $5 \times 10^{16} \mathrm{D}^{+}$jons $/ \mathrm{cm}^{2}$ is also included in figure 8 to indicate the enhancement of hydride peaks due to irradiation. The hydride peak positions at 20 values of $35 \cdot 90,41 \cdot 00,43 \cdot 05$ and $59 \cdot 30$ are indexed to be (101), (110), (002) and (200) peaks of epsilon hydride tetragonal phase. This is in contrast to the observation of Sheft et al (1976) who have indexed their X-ray results to fcc structure. The formation of titanium deuteride at the surface of titanium and not at the peak of the implantation profile is in agreement with the conclusion of neutron yield experiments. This perhaps is due to the displacement damage produced during deuterium implantation which is highest in this region rendering the deuteride precipitates unstable. This coupled with the radiation enhanced mobility of deuterium make deuterium to migrate towards the surface and form the deuteride phase there.

\section{Deuterium implantation effects in the subsurface layer of copper}

In contrast to $\mathrm{Ti}$, deuterium does not form any deuteride phase in $\mathrm{Cu}$, rather it is largely insoluble in $\mathrm{Cu}$. In this system the deuterium behaviour is expected to be dominated by 
deuterium defect interaction. We have investigated the evolution of trapped deuterium profile and surface topography in copper, implanted with $30 \mathrm{keV}$ deuterium ions to different doses by secondary ion mass spectrometry (SIMS) and scanning electron microscopy (SEM) respectively. The main results from these studies are listed below: (1) Deuterium ions implanted into copper at room temperature are trapped by lattice defects produced during implantation. The depth profile of deuterium at an implanted dose of $3 \times 10^{18} \mathrm{D}^{+}$ions $/ \mathrm{cm}^{2}$ matches with the damage profile obtained from TRIM Monte Carlo simulation code. (2) The depth profile of deuterium in $\mathrm{Cu}$ implanted to doses up to $1 \times 10^{19} \mathrm{D}^{+}$ions $/ \mathrm{cm}^{2}$ are shown in figures $9 \mathrm{a}$ and $\mathrm{b}$. It is seen that the depth profile of deuterium at an implanted dose of $6.5 \times 10^{18} \mathrm{D}^{+}$ions $/ \mathrm{cm}^{2}$ shows a broader profile compared to that observed at lower doses of $3 \times 10^{18} \mathrm{D}^{+}$ions $/ \mathrm{cm}^{2}$. On further implantation at a dose of $1 \times 10^{19} \mathrm{D}^{+}$ions $/ \mathrm{cm}^{2}$ the profile splits into two peaks (see figure 9a), a large peak at a depth of $664 \mathrm{~A}$ and the other at a depth of $3200 \mathrm{~A}$. (3) Scanning electron microscopy showed the presence of blisters at a dose of $3 \times 10^{18} \mathrm{D}^{+}$ions $/ \mathrm{cm}^{2}$ and higher. The mean size of the blister is $0.48 \mu \mathrm{m}$ at $3 \times 10^{18} \mathrm{D}^{+}$ions $/ \mathrm{cm}^{2}$ and $0.69 \mu \mathrm{m}$ at $1 \times 10^{19} \mathrm{D}^{+}$ions $/ \mathrm{cm}^{2}$. The observation that the deuterium profile matches with the damage profile produced during irradiation, is a consequence of the high deuterium mobility at room temperature and the strong trapping of deuterium at radiation produced vacancies and vacancy clusters. The splitting of a single implantation profile into two, has been reported during hydrogen implantation of $\mathrm{Al}$ (Kamada et al 1987) and in $\mathrm{He}$ implanted $\mathrm{Cu}$ (Terreault et al 1977) samples. In both these cases, the double peak appeared either at the critical dose for blister formation or before the blister formation. In contrast, we observe the profile splitting at much higher doses than the dose at which blisters were first seen. Our results indicate that the double peak structure is a result of the control of nucleation and growth of bubbles by the lateral compressive stress generated in the implanted layer of $\mathrm{Cu}$.

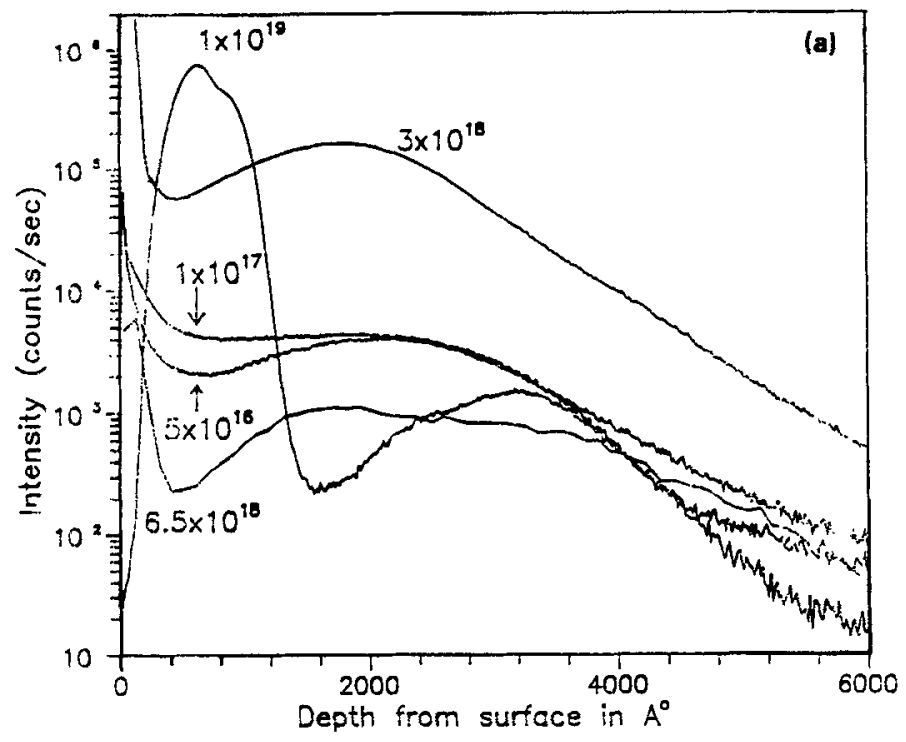

Figure 9. 


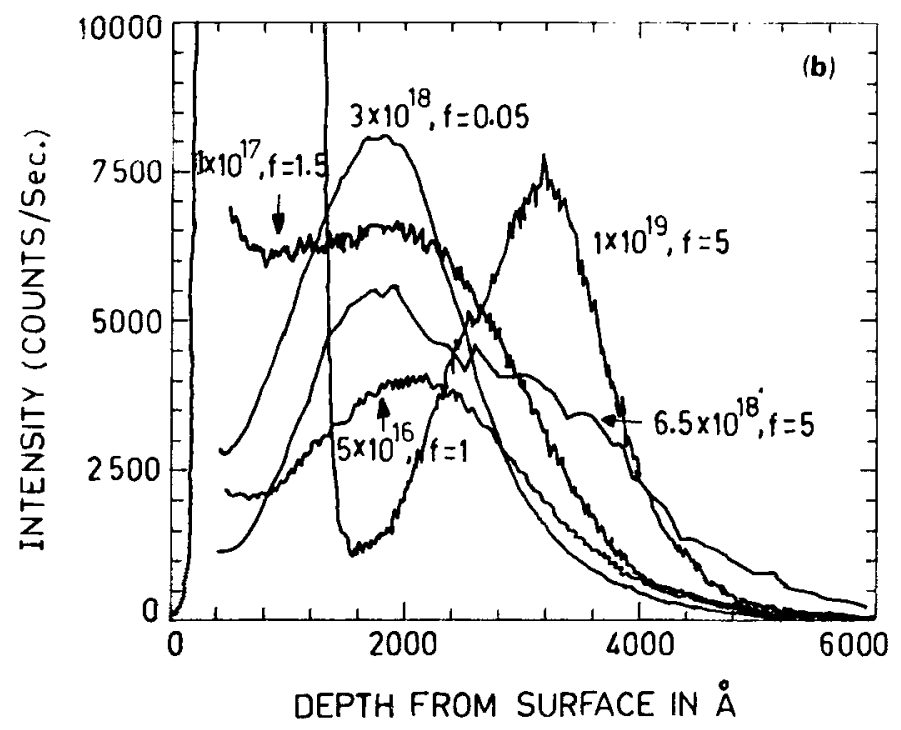

Figure 9. SIMS depth profile of deuterium from Cu samples implanted with $60 \mathrm{keV} \mathrm{D}_{2}^{+}$ions up to a dose of $1 \times 10^{19} \mathrm{D}^{+}$ions $/ \mathrm{cm}^{2}$ at a dose rate of $2.5 \times 10^{14} \mathrm{D}^{+}$ions $/ \mathrm{cm}^{2} / \mathrm{sec}$. (a) $\log$ scale and (b) linear scale. The depth profile at a dose of $6.5 \times 10^{18} \mathrm{D}^{+}$ions $/ \mathrm{cm}^{2}$ shows a broad profile compared to that observed at $3 \times 10^{18} \mathrm{D}^{+}$ions $/ \mathrm{cm}^{2}$ and splits into two peaks at dose of $3 \times 10^{19} \mathrm{D}^{+}$ions $/ \mathrm{cm}^{2}$. Note that the $y$-axis is arbitrary.

\section{Conclusion}

Dynamic and rion-equilibrum effects during deuterium implantation of $\mathrm{Cu}$ and Ti was studied. The main conclusions are: (i) During implantation, the deuteride phase in $\mathrm{Ti}$ forms at the surface and then grows inwards rather than forming at the peak of the implantation profile. (ii) The structure of Ti deuteride was found to be bct. (iii) The diffusion of deuterium in $\mathrm{Cu}$, during implantation at room temperature is via a vacancy mediated migration of deuterium trapped in vacancies. A diffusion constant around $5 \times 10^{-13} \mathrm{~cm}^{2} / \mathrm{sec}$ is estimated. (iv) The trapped deuterium profile in $\mathrm{Cu}$ splits into two peaks at a dose of $1 \times 10^{19} \mathrm{D}^{+}$ions $/ \mathrm{cm}^{2}$ which is attributed to the dynamics of bubble growth in the planted layers of $\mathrm{Cu}$.

\section{Acknowledgements}

The authors would like to thank their colleagues Dr. H K Sahu, Shri M C Valsakumar who have actively contributed in the analysis and measurements during the course of the experiments.

\section{References}

Kamada K, Sagara A. Kinoshita H and Takahashı H 1987 Rad. Effects 103119

Krauss A, Becker H W, Trautvetter W P, Rolfs C and Brand K 1987 Nucl. Phys. A150 465

Lengler B, Mantl S and Triftshauser W 1978 J. Phys. F: Metal Phys. 81691

Lindhards J and Scharff M 1961 Phys. Rev. 128124

Mansur L K, Lee E H, Maziasz P J and Rowcliffe A P 1986 J. Nucl. Mater. 141143633 
Mueller W M, Blackledge J P and Libowitz G G (eds) 1968 Metal hydrides (New York: Academic Press) Myers S M, Baskes M I, Birnbaum H K, Corbett J W, Deleo G G, Estreicher S K, Haller E E, Jena P, Johnson N M, Kircheim R, Pearton S J and Stavola M J 1992 Rev. Mod. Phys. 64559

Panigrahi $\mathrm{B} 1993$ Interaction of hydrogen and its isotopes with radiation effects in metals, Ph.D. Thesis, Indian Institute of Science, Bangalore (unpublished)

Panigrahi B, Krishan K, Sahu H K and Valsakumar M C 1992 Proc. Solid State Phys. Symp. 35C 233

Panigrahi B, Nair K G M, Sahu H K and Krishan K 1994 (to be published)

Sahu H K, Valsakumar M C, Panigrahi B, Nair K G M and Krishan K 1992 Pramana-J. Phys. 11739

Sheft Irving, Reiss Jr A H. Gruen D M and Peterson S W 1976 J. Nucl. Mater. 591

Terrault B, Martel J G, St-Jacques R G, Veilleux G, L'ecuyer J, Brassarrd C, Cardinal C, Descheres L and Labriie J P 1977 J. Nucl. Mater. 68334

Winter J, Phillips V, Samm U and Schweer B 1989 (eds) Proc. 8th international conference on plasma surface interactions in controlled fusion devices; $J$. Nucl. Mater. pp. 162 